\title{
Salud y conservación de la pava aliblanca Penelope albipennis (Cracidae)
}

Health and conservation of the white-winged turkey hen

Penelope albipennis (Cracidae)

Tatiana Cavero Aponte ${ }^{1}$

Hugo Samamé Beltrán²

http://dx.doi.org/10.21503/CienciayDesarrollo.2006.v6.04

RESUMEN

El bosque seco del Perú alberga grandes concentraciones de flora y fauna de distribución restringida. Una de las especies más amenazadas es la pava aliblanca Penelope albipennis, con una población estimada de 250 aves. Desde su redescubrimiento, se desarrolla en Lambayeque un Programa de conservación, que incluye cría en cautiverio, traslocación y reintroducción; y con la meta final de devolver a esta especie a su hábitat histórico, capaz de reproducirse y de mantener una población viable. Solo con un claro conocimiento de su salud y enfermedad, es posible tomar decisiones relacionadas al cautiverio y reintroducción. Para esto, el objetivo de identificar la exposición a enfermedades bacteriales y virales de importancia aviar, considerando también a las aves domésticas de los alrededores del zoocriadero. Los resultados muestran que las pavas han sido expuestas a enfermedades en bajo grado mientras que las aves domésticas lo han sido en alto grado, demostrando enfermedad activa. Esta zona es la mayor fuente de exposición hacia las pavas aliblancas. Estos resultados han permitido: 1) evaluar el estado actual de salud de las pavas y los riesgos en la población en cautiverio, 2) determinar acciones preventivas en el zoocriadero para evitar introducir patógenos al medio silvestre. El objetivo de cualquier reintroducción debe ser el de apoyar a las poblaciones silvestres sin dañarlas con la introducción de patógenos desde el cautiverio.

Palabras clave: bosque seco, pava aliblanca, aves domésticas, enfermedades, reintroducción.

\section{ABSTRACT}

The dry forest of Peru harbours great concentrations of flora and fauna of restricted distribution. One of the most threatened species is the white-winged turkey hen Penelope Albipennis, with a considered population of 250 birds. Since its rediscovery, a conservation Program is being developed in Lambayeque, which includes breeding in captivity, translocation and reintroduction; and with the final objective of giving back this species to its historic habitat, capable of reproducing and keeping a viable population. Only with a clear knowledge of its health and disease, it is possible to take decisions related to the captivity and reintroduction. To achieve this, the objective of identify the exposure to important bacterial and viral diseases of aviar importance, also considering the domestic birds that surround the zoo breeding

1. Universidad Alas Peruanas, Facultad de Ciencias Agropecuarias, Escuela Profesional de Medicina Veterinaria.

2. Asociación Cracidae Perú 
place. The results show that the turkey hens have been exposed to diseases in low degree whereas the domestic birds have been exposed in high degree, showing active disease. This area is the greater disease of exposure towards the turkey hens and the risks in the population in captivity have allowed. These results have allowed: 1) To evaluate the present state of turkey hen health and the risks in the population in captivity ,2)To determine preventive actions in the zoo breeding place to avoid the introduction of pathogens to the average wild. The aim of any reintroduction must be to support the wild populations without harming them with the introduction of pathogens from captivity.

Key words: dry forest, white-winged guan, domestic birds, diseases, reintroduction.

\section{INTRODUCCIÓN}

La región nor-oeste del Perú alberga grandes concentraciones de flora y fauna de distribución restringida, incluyendo 80 especies de aves endémicas y 21 amenazadas globalmente (Birdlife Internacional $\left.{ }^{1}\right)$. Pertenece al "Hotspot" TumbesChocó-Magdalena $(\mathrm{Cl})$ y es un Área Endémica de Aves (EBA), denominada Región Tumbesina, abarcando al menos 18 tipos de hábitat (Birdlife Internacional ${ }^{1}$ ). Según las Ecoregiones del Perú, el área pertenece al Bosque Seco Ecuatorial (Brack ${ }^{2}$ ); con un paisaje general llano, de bosques de algarrobo (Prosopis spp.), y montañas ondulantes de bosques deciduos que reverdecen con las lluvias. A lo largo de los ríos hay bosques de galería húmedos. Los cursos de agua son temporales; abundando con las lluvias y quedando reducidos a ojos de agua o "jagüeyes". El clima tiene dos épocas marcadas, una de lluvias y una seca. Las lluvias aumentan con evento El Niño, hasta los 4 años posteriores. Más del $80 \%$ de la precipitación medial anual $(100 \mathrm{~mm})$ es durante la época húmeda, siendo el resto del año de extrema aridez. Estos bosques representan unos de los más ricos y amenazados EBAs del planeta y están considerados con prioridad crítica de conservación por la severa pérdida de hábitat que enfrentan hoy en día (Birdlife Internacional, 2003).

Una de las especies más amenazadas de esta zona es la pava aliblanca Penelope albipennis (Birdlife Internacional, 2000); crácido ar- bóreo distribuido en una estrecha franja $\left(05^{\circ} 29^{\prime} \mathrm{S}\right.$ a $06^{\circ} 43^{\prime} S$ y $79^{\circ} 20^{\prime}$ a $79^{\circ} 50^{\prime} \mathrm{O}$ ), entre los 400 a 1200 msnm de la vertiente Occidental de los Andes. Fue descubierta por L. Taczanowsky en 1877 y considerada extinta casi todo el siglo XX, hasta su re-descubrimiento por J. O’Neill y G. Del Solar en 1977 (Ortiz \& Díaz, 1997). Actualmente, una población estimada de 250 aves sobrevive en su hábitat silvestre (Birdlife Internacional, 2000). Está incluida en el Apéndice I de CITES, categorizada como Críticamente amenazada (CR) según UICN, y como "En Peligro Crítico" por la legislación Peruana. La ley № 28049 la ha declarado "Ave de Interés Nacional", ofreciendo garantías legales para su protección (ACP, 2004).

Desde su redescubrimiento, se inició en Lambayeque el Programa de Conservación de la Pava Aliblanca; con actividades como cría en cautiverio en el zoocriadero Bárbara D’Achille, protección de hábitat, educación ambiental, investigación, así como traslocación, aclimatación en semi-cautiverio y reintroducción en el Área de Conservación Privada Chaparrí, donde hasta hace 20 años hubo pavas, pero se extinguieron. La meta a largo plazo es devolver esta especie a su hábitat histórico, capaz de reproducirse y de mantener una población viable (ACP, 2004).

Las pavas son dispersoras y depredadoras de las plantas, manteniendo estable su demografía. Esta especie es sensible a la caza y destrucción de hábitat, y ya que puede ser censada, 
puede usarse como especie indicadora de ecosistemas saludables (UICN Cracid Specialist Group, 2000). Asimismo, se le considera una especie bandera, ya que su conservación constituye una herramienta efectiva para la protección del bosque seco y, añadido al atractivo de especie endémica para el turismo especializado, genera la posibilidad de beneficios económicos para los pobladores locales, lo que los impulsa a ser guías, actuando paralelamente como guardianes de estos raros crácidos (Angulo, 2003). Sin embargo, las causas que la han puesto en peligro de extinción aún persisten. Mas aún, su fisiología reproductiva es una amenaza intrínsica. La tasa de natalidad es muy baja por el número pequeño de puesta (uno a tres, usualmente dos huevos) por temporada reproductiva, y su lento proceso de madurez (tres años) (Ortiz \& Díaz, 2997). Esta especie tiene conducta arisca y susceptibilidad al estrés, siendo la mayor causa de mortalidad (Todcidliwski et al. 1999; Cavero, 2005). El zoocriadero trabaja para maximizar su supervivencia y liberar los individuos más aptos, biológica y sanitariamente (Angulo, 2003).

La reintroducción comenzó el año 2000, formándose tres parejas, y todas intentaron reproducirse la siguiente temporada de cría. Aunque hubo depredación, el primer polluelo silvestre nació el año 2002 y hasta la fecha, quince polluelos han nacido en libertad, lo que constituye un éxito para la reintroducción y los objetivos del Programa. Se ha determinado en base a las distancias de dispersión, que es factible conectar la población reintroducida con las poblaciones silvestres aledañas (Angulo, 2003).

Ya que la reintroducción, y en el futuro cercano, la suplementación de las poblaciones silvestres, son las estrategias de manejo para esta especie, en la actualidad se ha tomado consciencia de que sólo con un claro conocimiento de la salud y enfermedad de las pavas es posible tomar decisiones relacionadas al cautiverio, la trasloca-

ción y la población silvestre. Para esto, el objetivo de Identificar la exposición a enfermedades bacteriales y virales de importancia aviar mediante pruebas serológicas en las pavas, pero considerando también a las aves domésticas de alrededores del zoocriadero, como referencia a las enfermedades estudiadas.

Excepto algunos análisis en las pavas a reintroducir, un mayor número de enfermedades en el resto de las aves, previo al presente estudio, no había sido evaluado.

\section{MATERIAL Y MÉTODO}

El 95\% $(n=86)$ de la población de pavas del zoocriadero Bárbara D'Achille (Olmos, Lambayeque) fue capturada manualmente para un examen físico y colecta de muestras sanguíneas a analizar: Mycoplasma gallisepticum, M. synoviae, Salmonella Enteritidis, S.Pullorum/ Gallinarum, Influenza Aviar, Enfermedad de Newcastle y Bursitis Infecciosa (Tabla 1).

Tabla 1. Patógenos específicos de las aves muestreadas* y las pruebas serológicas utilizadas para su detección. Olmos, Lambayeque, 2004.

\begin{tabular}{|c|c|}
\hline Enfermedad & Procedimiento \\
\hline Mycoplasma gallisepticum & $\begin{array}{l}\text { Aglutinación } \\
\text { en placa }\end{array}$ \\
\hline M. synoviae & $\begin{array}{l}\text { Aglutinación } \\
\text { en placa }\end{array}$ \\
\hline Salmonella Enteritidis & $\begin{array}{l}\text { Aglutinación } \\
\text { en placa }\end{array}$ \\
\hline \multicolumn{2}{|l|}{ Salmonella Pullorum } \\
\hline / S.Gallinarum & $\begin{array}{l}\text { Aglutinación } \\
\text { en placa }\end{array}$ \\
\hline \multicolumn{2}{|l|}{ Influenza Aviar ELISAa, } \\
\hline AGIDb & $\begin{array}{l}\text { Enfermedad de } \\
\text { New-castle }\end{array}$ \\
\hline
\end{tabular}

* CITES: Convención internacional de flora y fauna amenazada.

** UICN: Unión Internacional para la Conservación de la naturaleza. 


$\begin{array}{ll}\text { ELISA, HIC } & \begin{array}{l}\text { Bursitis } \\ \text { infecciosa }\end{array} \\ \text { ELISA } & \end{array}$

Pavas Aliblancas del zoocriadero Bárbara D’Achille y aves domésticas (pollos, pavos, patos) del caserío Las Pampas.

a: Ensayo inmunoabsorbente ligado a enzimas.

b: Agar Gel Inmuno Difusión.

c: Inhibición de la aglutinación.

\section{RESULTADOS}

De las pavas aliblancas muestreadas, 5 fueron pichones, 17 juveniles y 64 adultos; siendo 39 machos y 45 hembras, y desconociéndose el sexo de 2 aves. Las pavas examinadas estaban en buena condición física, excepto cinco: un macho con defectos congénitos $(1.16 \%)$, una hembra con una pata amputada (1.16\%) y tres hembras con infección al oído (3.49\%). Sólo la segunda ave no fue muestreada. Otras nueve aves (10.47\%), presentaron dedos torcidos o con deformidades en distintos grados (Tabla 2).

Tabla 2. Problemas encontrados en la población muestreada $(n=86)$ de pavas aliblancas. Zoocriadero Bárbara D’Achille, Olmos, Lambayeque, 2004.

\begin{tabular}{lr}
\hline Otros problemas & Número $(\%)$ \\
\hline Defectos congénitos & $1(1,16 \%)$ \\
Infecciones de oído & $3(3,49 \%)$ \\
Pata amputada & $1(1,16 \%)$ \\
Deformidades patas & $9(10,47 \%)$ \\
TOTAL & $14(16,28 \%)$
\end{tabular}

Con respecto a algunas muestras, la cantidad de sangre obtenida fue insuficiente, y otras se contaminaron, por lo que los números totales son en algunos casos menores.

No se encontraron evidencias de hemoparásitos en los frotices sanguíneos. Todas las pavas resultaron negativas a Mycoplasma gallisepticum.
Sólo 1 de $86(1,2 \%)$ resultó positiva a M.synoviae. De 83 aves, $8(9,6 \%)$ reaccionaron a Salmonella Enteritidis y 6 de $79(7,6 \%)$ a S.Pullorum/GaIlinarum. Todas las pavas resultaron negativas a Influenza Aviar. De 79 aves, una (1,3\%) resultó positiva a Newcastle con ELISA y posteriormente negativa con $\mathrm{HI}$; y otras $2(2,5 \%)$ resultaron positivas a Bursitis Infecciosa. De 85 pavas, $2(2,4 \%)$ resultaron con infección mixta.

En contraste, las aves domésticas presentaron sintomatología clínica diversa respiratoria, digestiva y neurológica, entre otros signos generales de enfermedad. Los resultados mostraron 15 de $24(62,5 \%)$ aves positivas a Mycoplasma gallisepticum, 12 de 16 (75\%) a M. synoviae, 3 de 17 $(17,6 \%)$ a Salmonella Enteritidis y 2 de $17(11,8 \%)$ a S.Pullorum/Gallinarum. Asimismo, 15 de 25 aves $(60 \%)$ resultaron positivas a Influenza Aviar con ELISA y posteriormente negativas con AGID; 15 de $25(60 \%)$ a Newcastle y 7 de $21(33,4 \%)$ a Bursitis Infecciosa. De 25 aves, 20 (76\%) resultaron con infección mixta (Figura 1).

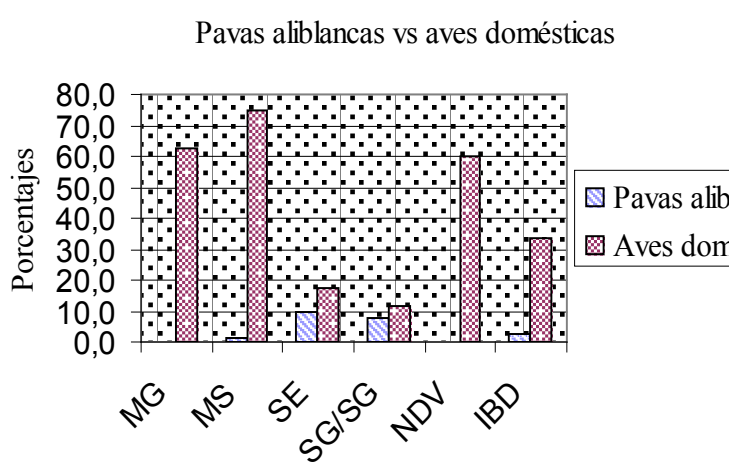

Figura 1. Agentes patógenos estudiados

Figura 1. Resultados comparativos de exposición a patógenos específicos en pavas aliblancas y aves domésticas. Olmos, Lambayeque, 2004.

\section{DISCUSIÓN}

La zona con aves domésticas no es ajena a otras realidades rurales, donde la forma de vida es la agricultura y crianza casera de animales para consumo. Éstos se procuran su propio 
alimento y viven en condiciones sanitarias muy precarias. La extrema sequedad del bosque seco afecta la abundancia de forraje y así, los parámetros productivos son los mínimos; favoreciendo la presentación y mantenimiento de agentes infecciosos. Los altos porcentajes de aves positivas y títulos encontrados, sumados a los reportes de mortalidades y sintomatología clínica, sugieren que, de ser evaluadas para más enfermedades, probablemente también resulten positivas en alta proporción.

El paisaje epidemiológico encontrado alrededor de las pavas demuestra que los reservorios de las enfermedades estudiadas y otras potencialmente peligrosas están muy cerca al zoocriadero. Esta cercanía hace esta zona la de mayor influencia negativa sobre el zoocriadero, que, aunque con porcentajes bajos, al estar rodeado por una zona de riesgo, se convierte también en una zona de riego (Cavero, 2005),

La seropositividad indica que ha habido una infección pasada o presente, y ya que los animales subclínicos, crónicamente infectados, son comunmente seronegativos, es posible que más pavas en este estudio estén infectadas. Todos estos agentes infecciosos tienen el potencial de afectar las dinámicas poblacionales. En cuanto a los hemoparásitos, ya que estos no siempre son encontrados en los eritrocitos circulantes de los animales infectados, los frotices negativos no indican ausencia de infección (Karesh et al. 1997). El hecho de que las pavas reintroducidas se agrupan y procrean, indica que las enfermedades de transmisión vertical (Salmonella, Mycoplasma) son de consideración importante. Salmonella y Newcastle también tienen implicancia en la salud pública. A pesar de que los patógenos estudiados han causado problemas en la avifauna de otras regiones del mundo (Drew, 2003; Friend \&Franson, 1999; Silvino, 1993), es difícil aun predecir la extensión de los daños en la población de pavas aliblancas. Es importante considerar que muchas aves endémicas de este ecosistema pueden sucumbir ante el contacto con patógenos aviares, como ha sido descrito en otras localidades (PIERCE, 2003; Jiménez, 2003). Las pavas reactoras positivas deben salir del programa de reproducción y/o reintroducción y ser evaluadas con pruebas más específicas (Charlton, 2000; Hagen et al. 2002). Las investigaciones de salud continúan.

Los resultados preliminares con ELISA para Influenza aviar y Newcastle permitieron acceder a las autoridades del Servicio Nacional de Salud Animal, SENASA. El hecho de que haya involucramiento del SENASA favorece directamente la protección hacia las pavas así como el bienestar de la población de Las Pampas.

En general, existe un gran debate acerca del rol de la crianza en cautiverio y la reintroducción en la conservación de especies amenazadas. Las enfermedades son siempre citadas como la razón principal para no conducir liberaciones desde el cautiverio. Sin embargo, hay circunstancias donde estas prácticas son las técnicas de manejo viables o la única alternativa (Brightsmith et al., 2005). El objetivo de cualquier reintroducción debe ser el de apoyar a las poblaciones silvestres sin dañarlas con la introducción de patógenos desde el cautiverio (IUCN Conservation Breeding Specialist Group, 2003). Por este motivo, ningún programa viable de reintroducción debe ser diseñado sin considerar los aspectos de bioseguridad de la especie involucrada (IUCN Veterinary Specialist Group, 2000). Es más fácil establecer un cordón sanitario y continuar con los estudios, que adoptar medidas de emergencia.

La prevención exitosa requiere que las acciones se basen en el concepto de que las enfermedades son generalmente un resultado más que una causa; siendo las condiciones ambientales usualmente la causa básica, asociadas a la emergencia, persistencia y diseminación de enfermedades; y por lo tanto deben ser claramente identificadas (Wikelski et al., 2004). Los documentos que registran las amenazas en los crácidos (CAMP, 
1994; IUCN Cracid Specialist Group, 2000), incluida la pava aliblanca, no consideran a las enfermedades como amenaza de esta pequeña población de aves.

La posibilidad de introducción de enfermedades desde las aves domésticas hacia las pavas es considerada alta; en consecuencia, son causa suficiente para que las enfermedades sean consideradas como una amenaza latente, próxima y causante de efectos deletéreos en esta especie. Mas aun, la tendencia de la población silvestre va en declive (Birdlife Internacional, 2003) y la población más numerosa ex situ está en el zoocriadero Bárbara D'Achille, albergando casi 1/3 de la población total estimada. Paradójicamente, la sensibilidad al estrés de las pavas se compensa con la aparente resistencia a enfermedades (Tocidlowdski et al., 1999; Tello \& Pedraglio, 1998); sin embargo estas pueden afectar de manera subclínica. Asimismo, este estudio ha sido realizado en época seca y un cambio ambiental tan radical como un evento El Niño puede hacer que estos desafíos y la susceptibilidad sean mucho mayores. Además, se desconoce si la mortalidad por enfermedades es mayor a la natural.

Los cambios continuos en el paisaje debido a las demandas humanas continuarán alterando las distribuciones geográficas y agregaciones de avifauna en una manera que facilitarán la emergencia de enfermedades y su diseminación entre aves silvestres (Wikelski et al., 2004). La visión frente a las enfermedades puede desafiar los puntos de vista de aquellos involucrados en la conservación de la fauna silvestre, ya que ven a las amenazas de enfermedad como algo menor comparado a otras más obvias, como la pérdida de hábitat, las especies introducidas, etc., y su experiencia con estos temas más públicos los vuelve reacios a dirigir sus limitados recursos hacia la detección y monitoreos de enfermedades (IUCN Conservation Breeding Specialist Group, 2003). Sin embargo, resultados concretos ayudan a que estas percepciones sean revertidas y así las eva- luaciones de salud y análisis de riesgo sean considerados parte de las estrategias para salvar a esta y otras especies en peligro (Munson, 2003). La idea es reevaluar las amenazas a favor de la avifauna, resultando en un enfoque pro-activo más que reactivo (Wikelski et al., 2004). De esta forma, se procura no introducir agentes infecciosos al medio silvestre.

Con el Programa de recuperación de la pava aliblanca se tiene la oportunidad de aplicar las lecciones aprendidas en cuanto a control de enfermedades y prevención de otras partes del mundo. Muchos de los riesgos de salud pueden ser manejados, influyendo positivamente en la conservación de estas aves y contribuyendo con los grandes esfuerzos por más de 27 años por salvar a esta especie bandera del bosque seco del Perú.

\section{REFERENCIAS BIBLIOGRÁFICAS}

1. ACP - Asociación Cracidae Perú. (2003). www.pavaaliblanca.org.

2. Angulo F. . Re-introduction of the white-winged guan in north-west Peru. Re-introduction News. 23 2003, 19-20.

3. IUCN/SSC Conservation Breeding Specialist Group. Animal Movement and Disease Risk: a Workbook. Armstrong, D., JakobHoff, R. \& Seal, U. (Eds). Minnesota: IUCN. 2003

4. Birdlife Internacional. IUCN Red list of Threatened Species: Penelope albipennis. www.redlist.org 2000.

5. BirdLife International. BirdLife's online World Bird Database: the site for bird conservation. http://www.birdlife.org 2003.

6. Brack, A. Ecología de un País Complejo: Ecorregión del Bosque seco Ecuatorial. En: Dourojeanni, M. Gran Geografía del Perú. México DF: Océano 1988, 279 - 282. 
7. Brightsmith, D., Hilburn, J., Del Campo, A., Boyd, J., Frisius, M., Frisius, R., Janik, D. \& Guillen, F. The use of hand-raised psittacines for reintroduction: a case study of scarlet macaws (Ara macao) in Peru and Costa Rica. Biological Conservation. 2005 , 121, 3, 465-472.

8. CAMP. Conservation Assessment and Management Plan: Penelope albipennis Taxon Report. En: Plassé C. (Ed.). Tercer Simposio Intencional de la Familia Cracidae; Memorias; Houston Zoological Society 1994.

9. Cavero, T. Serología para patógenos específicos de Pavas aliblancas (Penelope albipennis) del zoocriadero Bárbara D’Achille, Olmos, Lambayeque, Perú. Tesis de grado para optar el título de Médico Veterinario. Universidad Alas Peruanas 2005.

10. Charlton, K.G. Antibodies to Selected Disease Agents in Translocated Wild Turkeys in California. J Wildl Dis. 2000, 36, 1, 1614.

11. Cl - Conservation International. http://www. biodiversityhotspots.org/xp/ 2004.

12. Hotspots/tumbes_choco .

13. Drew, M. Galliformes (Pheasants, Grouse, Quail, Turkeys, Chachalacas, Curassows, Hoatzins). En: Fowler, M. \& Millar, E. Zoo and wild animal medicine. (pp. 161-171). Philadelphia: Saunders 2003.

14. Friend, M. \& Franson, C. (Eds). Field Manual of Avian Diseases: general field procedures and diseases of birds. Madison: USGS, Biological Resources Division, National Wildlife Health Center 1999.

15. Hagen, C. A., Crupper, S. S., Applegate, R. D. \& Robel, R.J. Prevalence of Mycoplasma Antibodies in Lesser Prairie-Chicken Sera. Avian Diseases. 2002, 46, 3, 708-712.
16. IUCN/SSC Cracid Specialist Group. Curassows, Guans and Chachalacas: Status Survey and Conservation Action Plan for Cracids 2000-2004. Brooks, D. \& Strahl, S. (Eds). Cambridge: IUCN 2000.

17. Jiménez, G. Agentes Infecciosos Registrados en las Aves Silvestres de Galápagos. Ecuador: Fundación Charles Darwin 2003.

18. Karesh, W., Del Campo, A., Braselton, E., Puche, H. \& Cook, R. Health Evaluation of Free-Ranging and Hand-reared Macaws (Ara spp.) in Perú. J Zoo and Wildl Med. 1997, 28, 4, 368-377.

19. Munson, L. Development of acceptable risks within the context of limited resources. En: Animal Movement and Disease Risk: a Workbook. UICN/SSC Conservation Breeding Specialist Group. Minnesota: IUCN 2003.

20. Ortiz, E. \& Díaz, V.R. Estudio de Campo y Reevaluación de la población de Pava Aliblanca (Penelope albipennis). En: Strahl, S.D., Beauion, S., Brooks, D.M., Begazo, J.A., Sedaghatkish, G. \& Olmos, F. (Eds.) The Cracidae: their Biology and Conservation. Hong Kong: Hancock House 1997.

21. PIERCE - Pacific Island Ecosystem Research Center. Impacts of Introduced Avian Diseases on the Decline and Extinction of Native Hawaiian Honeycreepers. PIERCE, USGS Biological Resources Division. http:// biology.usgs.gov/ pierce/index.htm 2003.

22. Silvino, C.Z. Natural Diseases of Free-Ranging Birds in South America. En: Fowler, M. Zoo and Wild Animal Medicine. (pp. 166172). Philadelphia: Sounders 1993.

23. Tello, A. \& Pedraglio, R. Posibilidad de la Crianza Comercial de Crácidos en Cautiverio. Strahl, S. (Ed.) Segundo Simposio Internacional de la Familia Cracidae; Memorias; Wildlife Conservation Society 1988. 
24. Tocidlowdski, M.E., Norton, T.M. \& Young, L.A. Medical management of curassows. En: American Association of Zoo Veterinarians: Proceedings; AAZV 1999.

25. Wikelski, M.J., Foufopoulos, H.V. \& Snell, H. Galápagos Birds and Diseases: Invasive Pathogens as Threats for Island Species. Ecology and Society. 2004, 9, 1-5.
26. Woodford, M.H. (Ed.). Quarantine and health screening protocols for wildlife translocation and release into the wild. Gland: IUCN SSP VSG, Paris: OIE, Switzerland: EAZWV, UK: Care for the Wild. 2000. 